\title{
The roles of IncRNA in hepatic fibrosis
}

\author{
Hu Peng ${ }^{1,3,4+}$, Lin-Yan Wan ${ }^{1,2,3+}$, Jia-Jie Liang ${ }^{1,3,4}$, Yan-Qiong Zhang ${ }^{1,3,4}$, Wen-Bing Ai $5^{*^{*}}$ \\ and Jiang-Feng $\mathrm{Wu}^{1,2,3,4,5^{*}}$ (1)
}

\begin{abstract}
Increasing evidence indicates that long non-coding RNAs (InCRNAs) regulate gene or protein expression; however, their function in the progression of hepatic fibrosis remains unclear. Hepatic fibrosis is a continuous wound-healing process caused by numerous chronic hepatic diseases, and the activation of hepatic stellate cells (HSCs) is generally considered to be a pivotal step in hepatic fibrosis. In the process of hepatic fibrosis, some IncRNAs regulates diverse cellular processes. Here are several examples: the IncRNA metastasis-associated lung adenocarcinoma transcript 1 (MALAT1) and liver fibrosis-associated IncRNA1 (Inc-LFAR1) promote HSC activation in the progression of hepatic fibrosis via the transforming growth factor- $\beta$ signaling pathway; the InCRNA HIF 1 alpha-antisense RNA 1 (HIF1A-AS1) and Maternally expressed gene 3 reduce HSC activation which are associated with DNA methylation; the IncRNA plasmacytoma variant translocation 1, Homeobox (HOX) transcript antisense RNA and MALAT1 promote HSC activation as competing endogenous RNAs (ceRNAs); the long intergenic non-coding RNA-p21 (IncRNA-p21) and Growth arrest-specific transcript 5 reduce HSC activation as ceRNAs. As we get to know more about the function of IncRNAs in hepatic fibrosis, more and more ideas for the molecular targeted therapy in hepatic fibrosis will be put forward.
\end{abstract}

Keywords: Long non-coding RNAs, Hepatic fibrosis, Hepatic stellate cell, TGF- $\beta$ signaling pathway, DNA methylation, ceRNA

\section{Background}

Hepatic fibrosis is a continuous wound-healing process that results in the dysregulation of extracellular matrix (ECM) proteins and the distortion of normal liver architecture [1]. Many chronic hepatic diseases, such as viral hepatitis, alcohol toxicity, drug abuse, metabolic syndrome, hereditary disorders of metabolism, autoimmune hepatitis and Clonorchis sinensis infection, lead to hepatic fibrosis and even cirrhosis [2], which is the primary stage of hepatic carcinoma, leading to one of the major causes of mortality in cancer worldwide.

Although extensive studies on hepatic fibrosis have been reported, their regulatory mechanisms are still partially understood. The activation of hepatic stellate cells (HSCs), the resident perisinusoidal cell type, is generally

\footnotetext{
*Correspondence: 1043642574@qq.com; wujiangfeng@ctgu.edu.cn

${ }^{\dagger} \mathrm{Hu}$ Peng and Lin-Yan Wan contributed equally to this work

${ }^{1}$ Medical College, China Three Gorges University, 8 Daxue Road, Xiling

District, Yichang 443002, China

${ }^{5}$ The Yiling Hospital of Yichang, 31 Donghu Road, Yi Ling District,

Yichang 443100, Hubei, China

Full list of author information is available at the end of the article
}

considered to be a pivotal step in hepatic fibrosis [3]. In normal hepatic tissue, HSCs with abundant vitamin A stores are quiescent. Following with hepatic injury of any etiology, the quiescent HSCs lose their stored vitamin A and trans-differentiate into fibrogenic myofibroblast-like cells. The activated HSCs are identified as proliferative cells that express ECM, and secrete profibrogenic mediators, thereby contributing to the fibrosis [4]. Therefore, the suppression of the HSC activation is regarded to be a potential therapeutic target for hepatic fibrosis.

Genome tiling arrays and cap analysis gene expression showed that non-protein coding RNAs (ncRNAs), which were considered to be "evolutionary junk" in the past, have more functions in transcription [5, 6]. Recently, a large number of ncRNA molecules have been identified by RNA microarrays and next-generation sequencing of transcriptomes [7]. NcRNAs are classified into two types based on their relative sizes. Those less than 200 nucleotides (nt) are called small or short non-coding RNAs, while those longer than $200 \mathrm{nt}$ are called long non-coding RNAs (lncRNAs) [8]. LncRNAs are considered to play roles in physiological conditions as well as in 


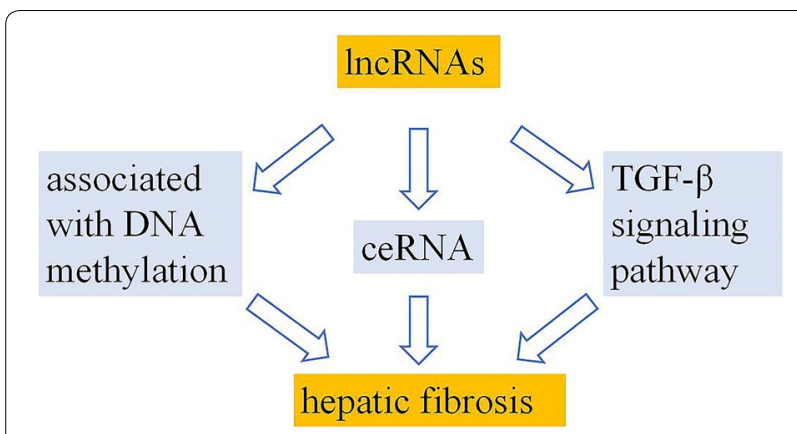

Fig. 1 It is the summary of the mechanism of IncRNAs that regulate hepatic fibrosis through TGF- $\beta$ signaling pathway, DNA methylation and ceRNA in this review

several human diseases, including cancer, metabolic diseases, cardiovascular diseases and so on [9]. Increasing evidence has suggested that lncRNAs regulate gene or protein expression by coordinating epigenetic, transcriptional, or post-transcriptional processes [10]. But the function of lncRNAs in hepatic fibrosis remains elusive.

The goal of this review is to summarize the roles of lncRNAs in hepatic fibrosis, including the regulation on transforming growth factor (TGF)- $\beta$ signaling pathway, DNA methylation and competing endogenous RNAs (ceRNAs) (Fig. 1) according to current knowledge. It will illustrate some important information for the treatment hepatic fibrosis and present novel guidance in future researches.

\section{The interaction of IncRNAs and the transforming growth factor (TGF)- $\beta$ signaling pathway in hepatic fibrosis}

TGF- $\beta$ is a key regulator of liver physiology and pathology during the process of initial liver injury-inflammation-fibrosis [11]. TGF- $\beta 1$, a potent fibrogenic cytokine from the autocrine or paracrine pathway, is a crucial signal that promotes HSC activation [12]. Some studies have shown that lncRNAs interact with the TGF- $\beta$ signaling pathway to promote HSC activation and then induce hepatic fibrosis.

Metastasis-associated lung adenocarcinoma transcript 1 (MALAT1) which is located in human chromosome 11q13.1 (mouse chromosome 19qA), also known as nuclear-enriched abundant transcript 2 (NEAT2), is a widely expressed lncRNA, and was firstly identified through subtractive hybridization in stage I of non-small cell lung cancer $[13,14]$. A growing number of evidence indicated that MALAT1 was closely related to various pathological processes, including diabetes complications and hepatic carcinoma [15], and could influence the progression of hepatic fibrosis by repressing the expression and function of silent information regulator 1 [SIRT1, a
Nicotinamide adenine dinucleotide (NAD)-dependent class III protein deacetylase] [16]. As one of the best characterized deacetylase enzymes, SIRT1 can protect cultured cells against metabolic, geneotoxic, hypoxic, and heat stress by deacetylating a number of key transcription factors [17], while it can induce the deacetylation of Smad3 (a downstream mediator of TGF- $\beta$ signaling pathway) and weaken the ability of Smad3 binding to the promoter of fibrogenic genes, such as collagen type I gene promoters, which means that the activation of SIRT1 attenuates TGF- $\beta$ signaling and then reduces TGF- $\beta$-stimulated collagen expression $[18,19]$. In summary, MALAT1 can promote the HSC activation through blocking the SIRT1 mediated inhibition of TGF- $\beta$ signaling pathway in the progression of hepatic fibrosis (Fig. 2a). What's more, MALAT1 is also reported that it acts as a competing endogenous RNA for miR-101b to regulate RAS-related C3 botulinum substrate 1 (Rac1) and contributes to hepatic fibrosis [20] (Fig. 4).

The liver fibrosis-associated lncRNA1 (lnc-LFAR1) is a $734 \mathrm{nt}$ transcript and it was originally identified as a liver-enriched lncRNA in fibrotic liver of mice. The lncLFAR1 of mice is located in chromosome 4q25, and it adjacents to the CYP2U1 and HADH genes which are the same as human [21]. ALGGEN-PROMO and JASPAR software analysis have shown that there are three potential Smad2/3 binding sites (SBE) in the promoter of lnc-LFAR1, which means that Smad2/3 can bind to

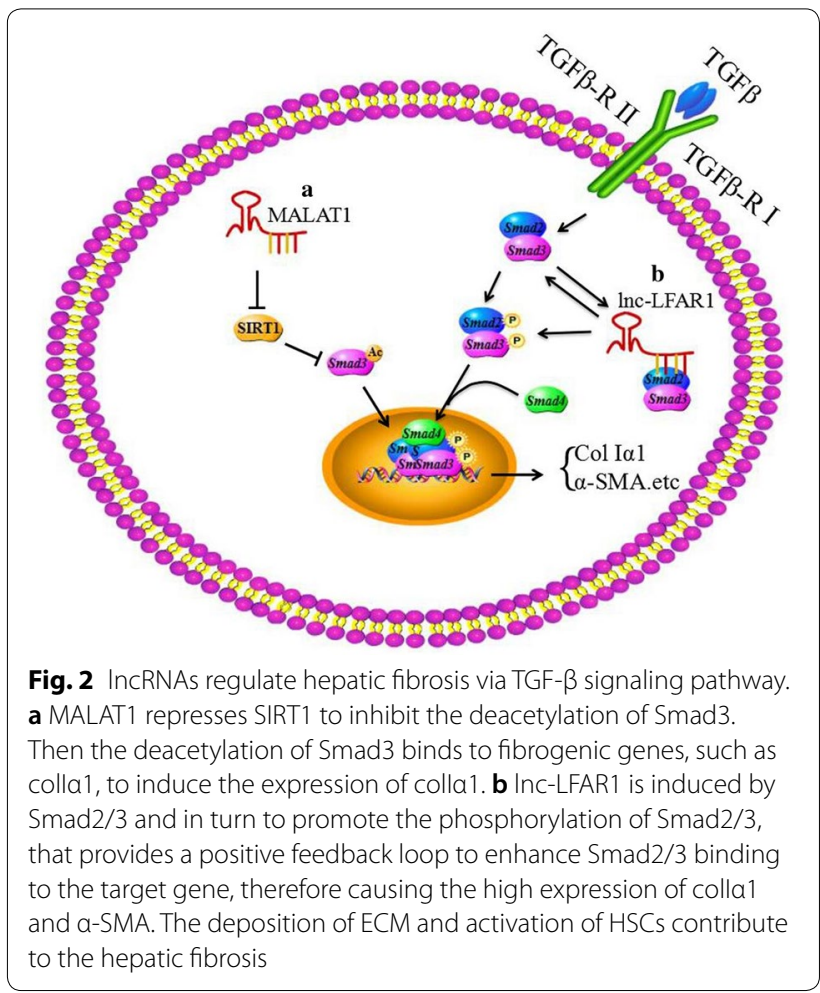


the promoter of lnc-LFAR1 to increase its expression. Furthermore, the lnc-LFAR1 in turn up regulates the expression of Smad2/3 and promotes Smad2/3 phosphorylation in liver fibrogenesis [21]. The phosphorylation of Smad2/3 promotes its nuclear translocation and the ability binding to the target promoters, such as collagen type I gene [22]. All in all, lnc-LFAR1 induces the activation of HSCs to promote hepatic fibrosis by interacting with TGF- $\beta$ signaling pathway (Fig. $2 b$ ).

\section{IncRNA associates with DNA methylation to inhibit the activation of HSCs in hepatic fibrosis}

DNA methylation is a type of epigenetic modification in mammals and involves in numerous biological processes, including transposable element silencing, genomic imprinting and $X$ chromosome inactivation [23]. The process of DNA methylation is regulated by methyltransferases, for examples, DNA methyltransferases (DNMT1, DNMT3a, DNMT3b) induce de novo methylation and ten-eleven translocation methylcytosine dioxygenase (TET) family member enzymes (TET1, 2 and 3) induce DNA demethylation re-activated or re-expressed silenced genes. DNMTs and TETs are critical in the cycle of DNA methylation and demethylation [24]. Advancing studies indicate that DNMTs and TETs play significant roles to change 5-methylcytosine and 5-hydroxymethylcytosine during HSC transdifferentiation to myofibroblast-like cells [25]. Increasing evidence shows that several lncRNAs are associated with DNA methylation to inhibit the activation of HSCs in hepatic fibrosis.

Maternally expressed gene 3 [MEG3, which is also known as gene trap locus 2 (GTL2)], is a lncRNA with the length of $1.6 \mathrm{~kb}$ nucleotides. It is a part of the DLK1-MEG3 imprinting locus and located at human chromosome $14 \mathrm{q} 32$ and at mouse distal chromosome 12 [26]. It is expressed in virous human tissues and acts as a tumor suppressor [27]. Recently, the loss of MEG3 expression has been gradually proved in various types of human cancers, such as hepatic cancer, gastric cancer, lung cancer, glioma, cervical cancer, bladder cancer [28-34]. On the one hand, MEG3 can selectively regulate p53 target gene expression resulting in the accumulation of $\mathrm{p} 53$ protein, and leading to cell growth inhibition [35]; on the other hand, MEG3 activates $\mathrm{p} 53$, and then intervenes in the p53-dependent mitochondrial apoptosis pathway to increase mitochondrial cytochrome $\mathrm{c}$ release and to culminate in direct caspase activation [35]. The expression of MEG3 was negatively correlated with the differentially methylated regions (DMRs) hypermethylation level, suggesting that DNA methylation plays an important role in silencing the MGE3 gene [28]. DNA methyltransferase 1 (DNMT1) can maintain methylation pattern on the daughter strand after DNA methylation and contribute to hypermethylation of MEG3 gene promoter and decrease the expression of MEG3 [30]. MEG3 could activate p53 to cause caspase-3-dependent apoptosis and reduce the expression of alpha-1 type I collagen (colI $\alpha 1)$ and $\alpha$-smooth muscle actin ( $\alpha$-SMA) in activeted HSCs induced by TGF- $\beta 1$ [36]. It is indicated that MEG3 plays a critical role in HSC activation and hepatic fibrogenesis (Fig. 3). Therefore, it reveals that high-expression of MEG3 are potentially regarded as a novel therapeutic target for treating liver fibrosis.

The lncRNA HIF 1 alpha-antisense RNA 1 (HIF1AAS1) was initially reported in human kidney cancers and it is located on chromosome 14, with $2100 \mathrm{nt}$ [37]. It has been demonstrated that the HIF1A-AS1 involves in the proliferation and apoptosis of vascular smooth muscle cells and the vascular endothelial cells $[38,39]$, and it is also related with the process of the non-small cell lung cancer and the colorectal carcinoma [40, 41]. HIF1A-AS1 acts as an inhibitor or an activator of cell proliferation and apoptosis depending on its binding partners and cell types. As one of the ten to eleven translocation (TET) family members, TET3 can catalyze 5-methylcytosine (5-mC) demethylate to 5-hydroxymethylcytosines (5-hmCs), which leads to cancer suppression [42]. In hepatic fibrosis, TET3 promotes the activation of HSCs through suppressing the expression of 1ncRNA1A-AS1 [43]. Thus, lncRNA HIF1A-AS1 interacts with the partner TET3 associated with DNA methylation to inhibit the activation of HSCs. These indicate that the up-regulation of lncRNA HIF1A-AS1 may be a potential therapy pathway for hepatic fibrosis (Fig. 3).

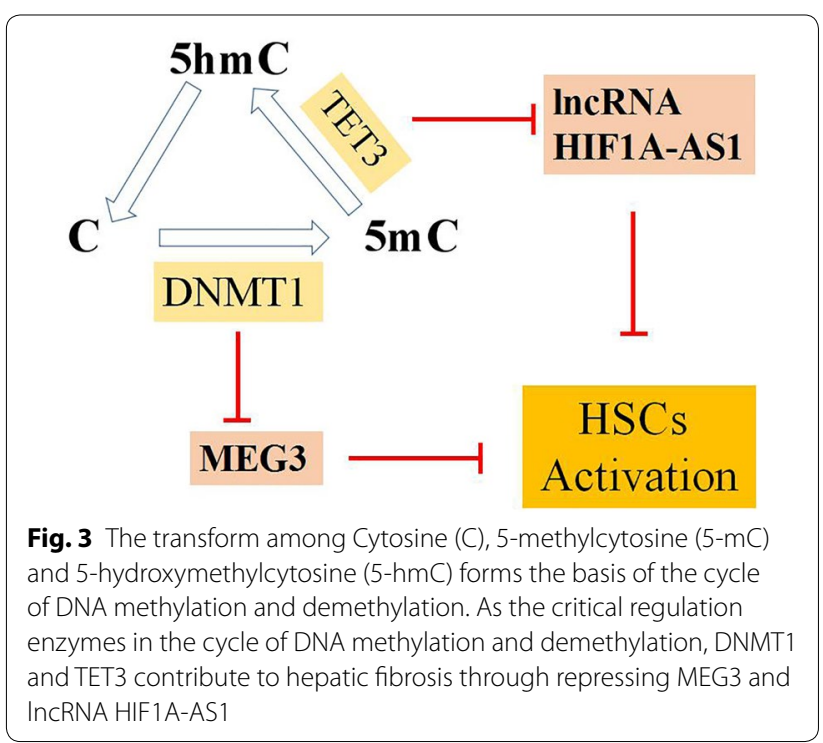




\section{IncRNAs act as ceRNAs in hepatic fibrosis}

MicroRNAs (miRNAs) pair with miRNA response elements (MREs) on target RNA transcripts resulting in degradation or translational repression of the target transcripts [44]. A competing endogenous RNA (ceRNA) is a endogenous origin transcript targeted by a miRNA that sequesters the activity of the bound miRNA, effectively de-repressing other targets of that miRNA [45]. LncRNAs have been gained substantial attention as ceRNAs to sponge miRNAs to consequently modulate the derepression of miRNA targets, thereby protecting their target mRNAs [46]. Recently, several studies have shown that lncRNAs act as ceRNAs which play an important regulatory role in the process of hepatic fibrosis.

The long intergenic non-coding RNA-p21 (lncRNAp21), which resides $15 \mathrm{~Kb}$ upstream of the gene encoding the critical cell cycle regulator Cdkn1a (also known as p21), contains two exons comprising $3.1 \mathrm{~Kb}$ [47]. LncRNA-p21 functions as a downstream transcriptional repressor in the p53 pathway via activating p53 to promote apoptosis [48]. It has been reported that lncRNAp21 has been deregulated in various human diseases, such as skin tumors, prostate cancer and hepatocellular carcinoma [49-51]. It also acts as a tumor suppressor in cancers, but the mechanism of the process remains unclear. LncRNA-p21 directly binds target mRNA to regulate the translation as a post-transcriptional inhibitor [52], while it also acts as a locus-restricted coactivator for p53-mediated p21 expression in regulating the G1/S checkpoint [53]. Furthermore, it has been proposed that the lncRNA-p21 is also able to regulate gene expression by directing the chromatin localization of protein binding partners [54]. As a tumor suppressor, the phosphatase and tensin homologue deleted on chromosome 10 (PTEN) is often deregulated in various cancers, and it is a direct target of miR-181b that has been reported in hepatic fibrosis [55]. Yu et al. demonstrated that the lncRNA-p21 enhanced PTEN expression through competitively binding miR-181b as a ceRNA and inhibited the activation of HSCs via PTEN/Akt pathway in hepatic fibrosis [56]. It is also reported that the lncRNA-p21 sponges miR-17-5p to inhibit WIF1 through Wnt/ $\beta$ catenin pathway resulting in suppression the HSC activation [57]. According to the research results above, we conclude that the lncRNA-p21 acts as a ceRNA to prevent the HSC activation in hepatic fibrosis.

Growth arrest-specific transcript 5 (GAS5) was initially discovered in a screen for potential tumor suppressor genes which expressed at high levels during growth arrest and it was originally isolated from mouse embryo NIH 3T3 cells using subtraction hybridization [58]. GAS5 has been reported as a tumor suppressor in some kinds of cancers, and it has been shown the GAS5 is involved in proliferation, apoptosis, and migration of tumor cells in breast cancer, gastric cancer and prostate cancer [59-61]. The GAS5 directly binds miR-21 to down-regulate its expression at exon 4 of GAS5 and negatively regulate the expression of miR-21 in hepatocellular carcinoma [62]. Moreover, it was also demonstrated that the GAS5 acts as ceRNA to control cardiac fibroblast activation and cardiac fibrosis by targeting miR-21 through PTEN/MMP-2 signaling pathway [63]. In hepatic fibrosis, the GAS5 through interacting with miR-222 to promote the expression of p27 protein, thereby inhibiting the activation and proliferation of HSCs [64].

Plasmacytoma variant translocation 1 (PVT1) in size of $>300 \mathrm{nt}$ is transcribed from a locus adjacent to the MYC locus on human chromosome 8q24 (mouse chromosome 15) [65]. Recently, the PVT1 is found to be up-regulated in a series of human tumors, such as hepatocellular carcinoma, ovarian cancer, malignant pleural mesothelioma, non-small lung cancer and renal cancer [66-70]. PVT1 was deemed as a mediator of ECM in the diabetic kidney [71], which suggested that the PVT1 might involve in fibrosis. Epithelial-mesenchymal transition (EMT) process is considered as a key event in the activation of HSCs and hepatic fibrosis via activating Hedgehog $(\mathrm{Hh})$ signaling pathway [72]. Patched1 (PTCH1), a member of Hh family, is also a negative regulator of Hh pathway. PVT1 can indirectly enhance PTCH1 methylation and down-regulate the expression of PTCH1 via competitively binding miR152. Therefore, the PVT1 may serve as a ceRNA for miR-152 through Hh pathway to regulate the activation of HSC in hepatic fibrosis [65].

Homeobox (HOX) transcript antisense RNA (HOTAIR) is a $2158 \mathrm{nt}$ lncRNA that locates to a boundary of the HOXC locus, one of the four chromosomal loci (HOX A to D) containing the clustered HOX genes [73]. Accumulating studies have indicated that HOTAIR is up-regulated in multiple cancers, including breast cancer, lung adenocarcinoma, renal cell carcinoma, pancreatic cancer, hepatocellular carcinoma [74-78]. MiR-29b can up-regulate the expression of PTEN via DNMT3b to suppress liver fibrosis [79]. The HOTAIR acts as a ceRNA to sponge miR-29b and then attenuates DNMT3b, leading to enhancement of PTEN methylation that contributes to liver fibrosis [80].

Whether ceRNA is an inhibitor or an activator to HSC activation depends on the spongy of miRNAs (Fig. 4). The model of how lncRNA works as ceRNA to sponge miRNAs may be widely accepted in (Fig. 5). The binding sites between lncRNA and miRNA shown in this review are displayed in the table (Table 1 ). 
Peng et al. Cell Biosci

(2018) 8:63

Page 5 of 8
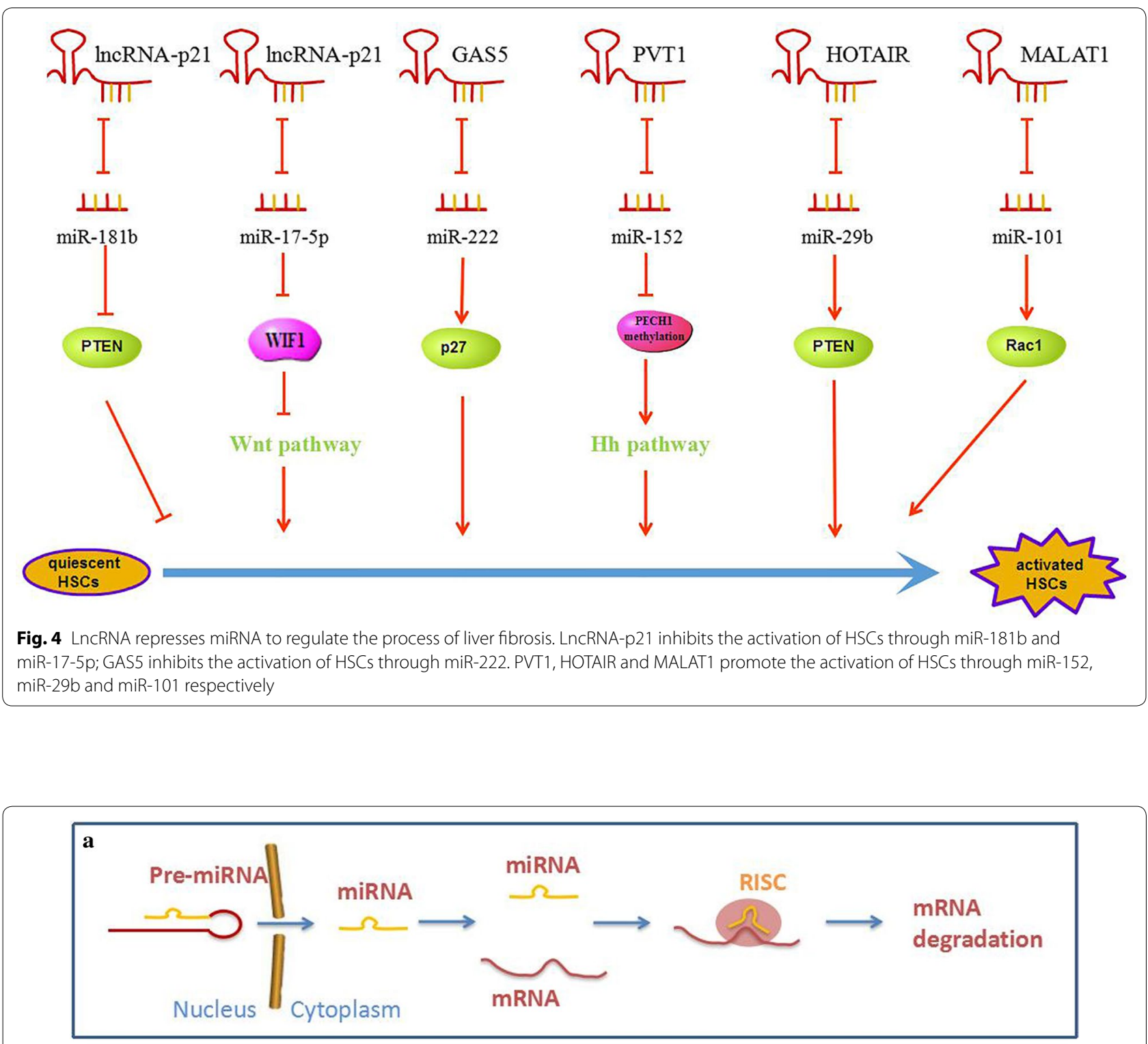

b

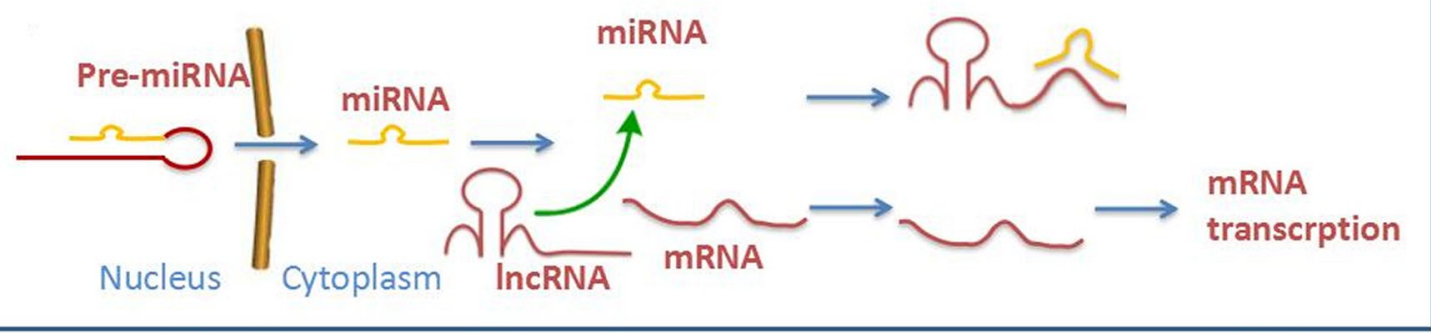

Fig. 5 a The pre-miRNA gets out from the nucleus to be the mature miRA. The mature miRA incorporates into the RISC and the target mANA to the target ERNA. $\mathbf{b}$ The activity of the miRNAs is inhibited by the presence of IncRNAs, which act as ceRNAs by sharing common AREs. Low levels of available miRNAs for the target mANA translations 
Table 1 IncRNA as ceRNA in hepatic fibrosis

\begin{tabular}{|c|c|c|c|}
\hline IncRNA & miRNA & binding region & \\
\hline & miR-181b & GCCTGCTGACAGCCAGAGAGGGTA & lncRNA-p21 \\
\hline \multirow{5}{*}{$\operatorname{lncRNA-p} 21$} & & $:|| \quad|:|||||||:||:||$ & \\
\hline & & TGGGTGGCTGTCG-TTACTTACAA & miR-181b \\
\hline & miR-17-5p & TCGGTTGCATCATCTCCCAGCTTTG & lncRNA-p21 \\
\hline & & $::||||:$ & \\
\hline & & GATGGACGTG--ACATTCGTGAAAC & $\operatorname{miR}-17-5 p$ \\
\hline \multirow[t]{3}{*}{ GAS5 } & $\operatorname{miR}-21$ & ACAGGCATTAGACAGA-AAGCTG & GAS5 \\
\hline & & $::|||:||||||||| \mid:$ & \\
\hline & & AG-UUGUAGUCAGACUAUUCGAU & $\operatorname{miR}-21$ \\
\hline \multirow[t]{3}{*}{ PVT1 } & miR-152 & CCAG---CCTGTTCTGCCCTGG & PVT1 \\
\hline & & \|\|\|\|\|\| & \\
\hline & & GGTTCAAGACAGTACGTGACT & $\operatorname{miR}-152$ \\
\hline \multirow[t]{3}{*}{ HOTAIR } & $\operatorname{miR}-29 b$ & TTTTTAAATTTCAACTTGGAGCTC & HOTAIR \\
\hline & & \|\|\|\||||||| $\mid$ & \\
\hline & & TTGTGACTAAAGTT-TACCACGAT & $\operatorname{miR}-29 b$ \\
\hline \multirow[t]{3}{*}{ MALAT1 } & $\mathrm{miR}-101 \mathrm{~b}$ & AGCCGAGTCCCTTTGCTGTGC & MALAT1 \\
\hline & & ||$|:||| \quad|:||| \mid:$ & \\
\hline & & TCG--ATAGTGTCATGACATG & $\operatorname{miR}-29 b$ \\
\hline
\end{tabular}

Only these IncRNAs that are described in this review are shown

\section{Conclusion}

Attention has been paid to lncRNA structure, function and evolution. Although there is a great interest in new lncRNAs, it is a scientific topic in the future. To explore new therapies of hepatic fibrosis, more lncRNAs have been found to be involved in the activation of HSCs.

MALAT1 and lncRNA-LFAR1 activate HSCs through the TGF- $\beta$ signaling pathway. Some lncRNAs will be discovered to induce the activation of HSCs via the TGF- $\beta$ signaling pathway in the future. Other signaling pathways, such as Wnt, NF-kB and Notch signaling pathway, are also related with the HSC activation. The lncRNA AC067945.2 down-regulates collagen expression in skin fibroblasts and it possibly correlates with the VEGF and Wnt signalling pathways [81]. Therefore, it is worthwhile to explore some new lncRNAs via other signaling pathways that take part in the activation of HSCs.

MEG3 and HIF1A-AS1 inhabit the HSC activation with DNA methylation. MEG3 and HIF1A-AS1 are repressed by DNMT1 and TET3 respectively. MEG3 causes the accumulation and activation of p53 that decreases proliferation and increases apoptosis of activated HSCs. The mechanism of HIF1A-AS1 inhabiting the HSC activation is still need to be explored. It is also possible that DNMT1 or TET3 regulates other lncRNAs in the process of hepatic fibrosis. Thus, the interaction between lncRNAs and other enzymes associated with methylation is also worth studying in hepatic fibrosis.

The last role of lncRNAs regulating the HSC activation is to be ceRNAs for miRNAs. PVT1, HOTAIR and
MALAT1 promote the activation of HSCs. LncRNA-p21 and GAS5 reduce the activation of HSCs. The miRNAs are inhibited by the lncRNAs which act as ceRNAs via sharing common MREs. The interaction between lncRNAs and miRNAs is not a one-to-one relationship, for example, lncRNA-p21 represses miR-181b and miR17-5p. In addition, lncRNA MIR100HG has been confirmed to encode miR-100, let-7a-2 and miR-125b-1. It is worth exploring whether any lncRNAs regulate miRcluster in liver fibrosis or not.

The differences in the expression of lncRNA between normal and hepatic fibrotic tissues not only imply that lncRNAs may take part in the progression of the hepatic fibrosis, but also suggest that IncRNAs may be the biomarkers for the clinical diagnosis of hepatic fibrosis. Moreover, it is possible that not only lncRNAs itself, but also both the binding proteins and the target genes will be new therapeutic targets, which may lead to the development of new anti-fibrosis treatments.

\begin{abstract}
Abbreviations
IncRNAs: long non-coding RNAs; ECM: extracellular matrix; HSCs: hepatic stellate cells; ceRNAs: competing endogenous RNAs; TGF- $\beta$ : transforming growth factor- $\beta$; MALAT1: metastasis-associated lung adenocarcinoma transcript 1; SIRT1: silent information regulator 1; Inc-LFAR1: liver fibrosis-associated InCRNA1; SBE: Smad2/3 binding sites; DNMT: DNA methyltransferases; TET: ten-eleven translocation methylcytosine dioxygenase; MEG3: maternally expressed gene 3; a-SMA: a-smooth muscle actin colla1: alpha-1 type I collagen; miRNAs: MicroRNAs; HIF1A-AS1: IncRNA HIF 1 alpha-antisense RNA 1; IncRNA-p21: long intergenic non-coding RNA-p21; PTEN: the phosphatase and tensin homologue deleted on chromosome 10; GAS5: growth arrestspecific transcript 5; PVT1: plasmacytoma variant translocation 1; HOTAIR: Homeobox (HOX) transcript antisense RNA; MREs: miRNA response elements.
\end{abstract}

\section{Authors' contributions}

HP and LYW contributed equally to this work and wrote the manuscript. JJL and YQZ gave some helps for this work. WBA and JFW revised and approved the article prior to its being submitted for publication. All authors read and approved the final manuscript.

\section{Author details \\ ${ }^{1}$ Medical College, China Three Gorges University, 8 Daxue Road, Xiling District, Yichang 443002, China. ${ }^{2}$ Digestive Medicine, The People's Hospital of China Three Gorges University, 31 Huti Subdistrict, Xi Ling District, Yichang 443000, Hubei, China. ${ }^{3}$ Institute of Organ Fibrosis and Targeted Drug Delivery, China Three Gorges University, 8 Daxue Road, Xiling District, Yichang 443002, China. ${ }^{4}$ Hubei Key Laboratory of Tumor Microenvironment and Immunotherapy, China Three Gorges University, 8 Daxue Road, Xiling District, Yichang 443002, China. ${ }^{5}$ The Yiling Hospital of Yichang, 31 Donghu Road, Yi Ling District, Yichang 443100, Hubei, China.}

\section{Acknowledgements}

We are thankful for the financial support of the National Natural Science Foundation of China (Grant Number: 81670555) and the Science Research Innovation Foundation of Graduate Student of China Three Gorges University (No. 2018SSPY106). We also thank Shi-Zhen Zhao and Shan-Bing Yin for English language polishing.

\section{Competing interests}

The authors declare that they have no competing interests.

Availability of data and materials Not applicable. 


\section{Funding}

The National Natural Science Foundation of China (Grant Numbers: 81670555) and the Science Research Innovation Foundation of Graduate Student of China Three Gorges University (No. 2018SSPY106) has given financial support.

\section{Publisher's Note}

Springer Nature remains neutral with regard to jurisdictional claims in published maps and institutional affiliations.

Received: 23 May 2018 Accepted: 22 November 2018

Published online: 06 December 2018

\section{References}

1. Wang J, et al. microRNA-29b prevents liver fibrosis by attenuating hepatic stellate cell activation and inducing apoptosis through targeting PI3K/ AKT pathway. Oncotarget. 2015;6:7325-38. https://doi.org/10.18632/ oncotarget.2621.

2. Wang $X$, et al. Proteomic identification of potential Clonorchis sinensis excretory/secretory products capable of binding and activating human hepatic stellate cells. Parasitol Res. 2014;113:3063-71. https://doi. org/10.1007/s00436-014-3972-z.

3. Seki E, Schwabe RF. Hepatic inflammation and fibrosis: functional links and key pathways. Hepatology. 2015;61:1066-79. https://doi. org/10.1002/hep.27332

4. Henderson NC, Iredale JP. Liver fibrosis: cellular mechanisms of progression and resolution. Clin Sci (Lond). 2007;112:265-80. https://doi. org/10.1042/cs20060242.

5. Kapranov P, Drenkow J, Cheng J, Long J, Helt G, Dike S, Gingeras TR. Examples of the complex architecture of the human transcriptome revealed by RACE and high-density tiling arrays. Genome Res. 2005;15:987-97. https ://doi.org/10.1101/gr.3455305.

6. Carninci $P$, et al. The transcriptional landscape of the mammalian genome. Science. 2006;309:1559-63. https://doi.org/10.1126/scien ce.1112014

7. Koh W, et al. Noninvasive in vivo monitoring of tissue-specific global gene expression in humans. Proc Natl Acad of Sci USA. 2014;111:7361-6. https://doi.org/10.1073/pnas.1405528111.

8. Beermann J, et al. Non-coding RNAs in development and disease: background, mechanisms, and therapeutic approaches. Physiol Rev. 2016;96:1297-325. https://doi.org/10.1152/physrev.00041.2015.

9. Harries LW. Long non-coding RNAs and human disease. Biochem Soc Trans. 2012;40:902-6. https://doi.org/10.1042/bst20120020.

10. Signal B, Gloss BS, Dinger ME. Computational approaches for functional prediction and characterisation of long noncoding RNAs. Trends Genet. 2016;32:620-37. https://doi.org/10.1016/j.tig.2016.08.004.

11. Dooley S, Dijke PT. TGF- $\beta$ in progression of liver disease. Cell Tissue Res. 2012;347:245-56. https://doi.org/10.1007/s00441-011-1246-y.

12. Yang JW, Hien TT, Lim SC, Jun DW, Choi HS, Yoon JH, Cho IJ, Kang KW. Pin 1 induction in the fibrotic liver and its roles in TGF- $\beta 1$ expression and Smad2/3 phosphorylation. J Hepatol. 2014;60:1235-41. https://doi org/10.1016/j.jhep.2014.02.004.

13. Hutchinson JN, et al. A screen for nuclear transcripts identifies two linked noncoding RNAs associated with SC35 splicing domains. BMC Genomics. 2007;39:1471-2164. https://doi.org/10.1186/1471-2164-8-39.

14. Ji P, et al. MALAT-1, a novel noncoding RNA, and thymosin beta4 predict metastasis and survival in early-stage non-small cell lung cancer. Oncogene. 2003;22:8031-41. https://doi.org/10.1038/sj.onc.1206928.

15. Liu JY, et al. Pathogenic role of IncRNA-MALAT1 in endothelial cell dysfunction in diabetes mellitus. Cell Death Dis. 2014;5:e1506. https://doi. org/10.1038/cddis.2014.466.

16. Wu Y, et al. Silent information regulator 1 (SIRT1) ameliorates liver fibrosis via promoting activated stellate cell apoptosis and reversion. Toxicol Appl Pharmacol. 2015;289:163-76. https://doi.org/10.1016/j.taap.2015.09.028.

17. Guarente L. Hypoxic Hookup. Science. 2009;324:1281-2. https://doi org/10.1126/science.1175679.

18. Sun $L$, et al. Transcriptional repression of SIRT1 by protein inhibitor of activated STAT 4 (PIAS4) in hepatic stellate cells contributes to liver fibrosis. Sci Rep. 2016;6:28432. https://doi.org/10.1038/srep28432.
19. Wei J, et al. The histone deacetylase sirtuin 1 is reduced in systemic sclerosis and abrogates fibrotic responses by targeting transforming growth factor $\beta$ signaling. Arthritis Rheumatol. 2015;67:1323-34. https:// doi.org/10.1002/art.39061.

20. Yu F, et al. MALAT1 functions as a competing endogenous RNA to mediate Rac1 expression by sequestering miR-101 b in liver fibrosis. Cell Cycle. 2015:24:3885-96. https://doi.org/10.1080/15384101.2015.1120917.

21. Zhang $K$, et al. The liver-enriched Inc-LFAR1 promotes liver fibrosis by activating TGF $\beta$ and Notch pathways. Nat Commun. 2017;8:1-16. https:// doi.org/10.1038/s41467-017-00204-4.

22. Meng XM, et al. Smad2 protects against TGF- $\beta / S$ mad3-mediated renal fibrosis. J Am Soc Nephrol. 2010;21:1477-87. https://doi.org/10.1681/ asn.2009121244.

23. Wu SC, Zhang Y. Active DNA demethylation: many roads lead to Rome. Nat Rev Mol Cell Biol. 2010;11:607-20. https://doi.org/10.1038/nrm2950.

24. $\mathrm{Wu} \mathrm{H}$, et al. The real culprit in systemic lupus erythematosus: abnormal epigenetic regulation. Int J Mol Sci. 2015;16:11013-33. https://doi. org/10.3390/ijms160511013.

25. Page A, et al. Hepatic stellate cell transdifferentiation involves genomewide remodeling of the DNA methylation landscape. J Hepatol. 2016;64:661-73. https://doi.org/10.1016/j.jhep.2015.11.024.

26. Miyoshi N, Wagatsuma H, Wakana S, Shiroishi T, Nomura M, Aisaka K, Kohda T, Surani MA, Kaneko-Ishino T, Ishino F. Identification of an imprinted gene, Meg3/Gtl2 and its human homologue MEG3, first mapped on mouse distal chromosome 12 and human chromosome 14q Genes Cells. 2000:5:211-20.

27. Zhou Y, Zhang X, Klibanski A. MEG3 noncoding RNA: a tumor suppressor. J Mol Endocrinol. 2012;48:R45-53. https://doi.org/10.1530/jme-12-0008.

28. Anwar SL, Krech T, Hasemeier B, Schipper E, Schweitzer N, Vogel A, Kreipe H, Lehmann U. Loss of Imprinting and Allelic Switching at the DLK1-MEG3Locus in Human Hepatocellular Carcinoma. PLoS ONE. 2012;7:e49462. https://doi.org/10.1371/journal.pone.0049462.

29. Sun $M$, et al. Downregulated long noncoding RNA MEG3 is associated with poor prognosis and promotes cell proliferation in gastric cancer. Tomor Biol. 2014;35:1065-73. https://doi.org/10.1007/s13277-013-1142-z.

30. Yan J, et al. MiR-148a regulates MEG3 in gastric cancer by targeting DNA methyltransferase 1. Med Oncol. 2014;31:879. https://doi.org/10.1007/ s12032-014-0879-6.

31. Lu KH, et al. Long non-coding RNA MEG3 inhibits NSCLC cells proliferation and induces apoptosis by affecting p53 expression. BMC Cancer. 2013;13:461. https://doi.org/10.1186/1471-2407-13-461.

32. Wang P, Ren Z, Sun P. Overexpression of the long non-coding RNA MEG3 impairs in vitro glioma cell proliferation. J Cell Biochem. 2012;113:186874. https://doi.org/10.1002/jcb.24055.

33. Qin R, et al. Long non-coding RNA MEG3 inhibits the proliferation of cervical carcinoma cells through the induction of cell cycle arrest and apoptosis. Neoplasma. 2013;60:486-92. https://doi.org/10.4149/ neo_2013_063.

34. Ying $L$, et al. Downregulated MEG3 activates autophagy and increases cell proliferation in bladder cancer. Mol BioSyst. 2013;9:407. https://doi. org/10.1039/c2mb25386k.

35. Zhou Y, et al. Activation of p53 by MEG3 non-coding RNA. J Biol Chem. 2007:282:24731-42. https://doi.org/10.1074/jbc.m702029200.

36. He Y, et al. Inhibitory effects of long noncoding RNA MEG3 on hepatic stellate cells activation and liver fibrogenesis. Biochim Biophys Acta. 2014;1842:2204-15. https://doi.org/10.1016/j.bbadis.2014.08.015.

37. Bertozzi D, et al. Characterization of novel antisense HIF-1 a transcripts in human cancers. Cell Cycle. 2011;10:3189-97. https://doi.org/10.4161/ cc.10.18.17183.

38. Wang S, et al. BRG1 expression is increased in thoracic aortic aneurysms and regulates proliferation and apoptosis of vascular smooth muscle cells through the long non-coding RNA HIF1A-AS1 in vitro. Eur J Cardiothorac Surq. 2015;47:439-46. https://doi.org/10.1093/ejcts/ezu215.

39. Wang J, et al. Clopidogrel reduces apoptosis and promotes proliferation of human vascular endothelial cells induced by palmitic acid via suppression of the long non-coding RNA HIF1A-AS1 in vitro. Mol Cell Biochem. 2015;404:203-10. https://doi.org/10.1007/s11010-015-2379-1.

40. Tantai J, Hu D, Yang Y, Geng J. Combined identification of long noncoding RNA XIST and HIF1A-AS1 in serum as an effective screening for non-small cell lung cancer. Int J Clin Exp Patho. 2015;7:7887-95. 
41. Gong W, Tian M, Qiu H, Yang Z. Elevated serum level of IncRNA-HIF1A-ASI as a novel diagnostic predictor for worse prognosis in colorectal carcinoma. Cancer Biomark. 2017;12:54. https://doi.org/10.3233/cbm-170179.

42. Hsu CH, et al. TET1 suppresses cancer invasion by activating the tissue inhibitors of metalloproteinases. Cell Rep. 2012;2:568-79. https://doi. org/10.1016/j.celrep.2012.08.030.

43. Zhang QQ, et al. TET3 mediates the activation of human hepatic stellate cells via modulating the expression of long non-coding RNA HIF1A-AS1. Int J Clin Exp Patho. 2014;11:7744-51.

44. Bartel DP. MicroRNAs: target recognition and regulatory functions. Cell. 2009;136:215-33. https://doi.org/10.1016/j.cell.2009.01.002.

45. Thomson DW, Dinger ME. Endogenous microRNA sponges: evidence and controversy. Nat Rev Genet. 2016;17:272-83. https://doi.org/10.1038/ nrg.2016.20.

46. Shi $\mathrm{X}$, et al. Long non-coding RNAs: a new frontier in the study of human diseases. Cancer Lett. 2013;339:159-66. https://doi.org/10.1016/j.canle t.2013.06.013.

47. Riley T, et al. Transcriptional control of human p53-regulated genes. Nat Rev Mol Cell Biol. 2008;9:402-12. https://doi.org/10.1038/nrm2395.

48. Huarte $\mathrm{M}$, et al. A large intergenic noncoding RNA induced by p53 mediates global gene repression in the p53 response. Cell. 2010;142:409-19. https://doi.org/10.1016/j.cell.2010.06.040.

49. Jiang YJ, Bikle DD. LncRNA profiling reveals new mechanism for VDR protection against skin cancer formation. J Steroid Biochem Mol Biol. 2014;144:87-90. https://doi.org/10.1016/j.jsbmb.2013.11.018.

50. Işın M, Uysaler E, Özgür E, Köseoğlu H, Şanlı Öner, Yücel Ömer B, Gezer Uğur, Dalay N. Exosomal IncRNA-p21 levels may help to distinguish prostate cancer from benign disease. Front Genet. 2015; 6:168. https:// doi.org/10.3389/fgene.2015.00168.

51. Ning Y, Yong F, Haibin Z, Hui S, Nan Z, Guangshun Y. LincRNA-p21 activates endoplasmic reticulum stress and inhibits hepatocellular carcinoma. Oncotarget. 2015;6:28151-63. https://doi.org/10.18632/oncot arget.4661.

52. Yoon J, et al. LincRNA-p21 suppresses target mRNA translation. Mol Cell. 2012:47:648-55. https://doi.org/10.1016/j.molcel.2012.06.027.

53. Dimitrova N, Zamudio JR, Jong RM, Soukup D, Resnick R, Sarma K, Ward AJ, Raj A, Lee JT, Sharp PA, Jacks T. LincRNA-p21 activates p21 In cis to promote polycomb target gene expression and to enforce the G1/S checkpoint. Mol Cell. 2014;54:777-90. https://doi.org/10.1016/j.molce 1.2014.04.025.

54. Fatica A, Bozzoni I. Long non-coding RNAs: new players in cell differentiation and development. Nat Rev Genet. 2013;15:7-21. https://doi. org/10.1038/nrg3606.

55. Zheng J, et al. Hepatic stellate cell is activated by microRNA-181 b via PTEN/Akt pathway. Mol Cell Biochem. 2015;398:1-9. https://doi. org/10.1007/s11010-014-2199-8.

56. Yu F, et al. Identification of a novel lincRNA-p21-miR-181b-PTEN signaling cascade in liver fibrosis. Mediators Inflamm. 2016;2016:1-10. https://doi. org/10.1155/2016/9856538.

57. Yu F, et al. LincRNA-p21 inhibits the Wnt/ $\beta$-catenin pathway in activated hepatic stellate cells via sponging MicroRNA-17-5p. Cell Physiol Biochem. 2017:41:1970-80. https://doi.org/10.1159/000472410.

58. Schneider C, King RM, Philipson L. Genes specifically expressed at growth arrest of mammalian cells. Cell. 1988;54:787-93.

59. Mourtada-Maarabouni M, et al. GAS5, a non-protein-coding RNA, controls apoptosis and is downregulated in breast cancer. Oncogene. 2009;28:195-208. https://doi.org/10.1038/onc.2008.373.

60. Sun $M$, et al. Decreased expression of long noncoding RNA GAS5 indicates a poor prognosis and promotes cell proliferation in gastric cancer. BMC Cancer. 2014;14:319. https://doi.org/10.1186/1471-2407-14-319.

61. Yacqub-Usman K, Pickard MR, Williams GT. Reciprocal regulation of GAS5 IncRNA levels and mTOR inhibitor action in prostate cancer cells. Prostate. 2015;75:693-705. https://doi.org/10.1002/pros.22952.

62. Zhang Z, et al. Negative regulation of IncRNA GAS5 by miR-21. Cell Death Differ. 2013;20:1558-68. https://doi.org/10.1038/cdd.2013.110.

63. Tao $\mathrm{H}$, et al. LncRNA GAS5 controls cardiac fibroblast activation and fibrosis by targeting miR-21 via PTEN/MMP-2 signaling pathway. Toxicology. 2017;386:11-8. https://doi.org/10.1016/j.tox.2017.05.007.
64. Yu F, et al. Long non-coding RNA growth arrest-specific transcript 5 (GAS5) inhibits liver fibrogenesis through a mechanism of competing endogenous RNA. J Biol Chem. 2015;290:28286-98. https://doi. org/10.1074/jbc.m115.683813.

65. Barsotti AM, et al. p53-dependent Induction of PVT1 and miR-1204. J Biol Chem. 2012;287:2509-19. https://doi.org/10.1074/jbc.m111.322875.

66. Wang F, Yuan JH, Wang SB, Yang F, Yuan SX, Ye C, Yang N, Zhou WP, Li WL, LiW, Sun SH. Oncofetal long noncoding RNA PVT1 promotes proliferation and stem cell-like property of hepatocellular carcinoma cells by stabilizing NOP2. Hepatology. 2014;60:1278-90. https://doi.org/10.1002/ hep.27239.

67. Liu E, Liu Z, Zhou Y. Carboplatin-docetaxel-induced activity against ovarian cancer is dependent on up-regulated IncRNA PVT1. Int J Clin Exp Pathol. 2015;4:3803-10.

68. Riquelme $\mathrm{E}$, et al. Frequent coamplification and cooperation between C-MYC and PVT1 oncogenes promote malignant pleural mesothelioma. J Thorac Oncol. 2014;9:998-1007. https://doi.org/10.1097/jto.0000000000 000202.

69. Yang YR, et al. Increased expression of the IncRNA PVT1 promotes tumorigenesis in non-small cell lung cancer. Int J Clin Exp Pathol. 2014;10:6929-35.

70. Wu Q, Yang F, Yang Z, Fang Z, Fu W, Chen W, Liu X, Zhao J, Wang Q, Hu X, Li L. Long noncoding RNA PVT1 inhibits renal cancer cell apoptosis by up-regulating Mcl-1. Oncotarget. 2017;8:101865-75. https://doi. org/10.18632/oncotarget.21706.

71. Alvarez ML, DiStefano JK. Functional characterization of the plasmacytoma variant translocation 1 gene (PVT1) in diabetic nephropathy. PLoS ONE. 2011;6:e18671. https://doi.org/10.1371/journal.pone.0018671.

72. Choi SS, et al. Leptin promotes the myofibroblastic phenotype in hepatic stellate cells by activating the hedgehog pathway. J Biol Chem. 2010;285:36551-60. https://doi.org/10.1074/jbc.m110.168542.

73. Rinn JL, et al. Functional demarcation of active and silent chromatin domains in human HOX loci by noncoding RNAs. Cell. 2007;129:1311-23. https://doi.org/10.1016/j.cell.2007.05.022.

74. Lu L, et al. Association of large noncoding RNA HOTAIR expression and its downstream intergenic $\mathrm{CpG}$ island methylation with survival in breast cancer. Breast Cancer Res Treat. 2012;136:875-83. https://doi.org/10.1007/ s10549-012-2314-Z

75. Yuanshun LIU, et al. Lentivirus-mediated silencing of HOTAIR IncRNA restores gefitinib sensitivity by activating Bax/Caspase-3 and suppressing TGF-a/EGFR signaling in lung adenocarcinoma. Oncol Lett. 2018;15:282938. https://doi.org/10.3892/ol.2017.7656.

76. Dasgupta P, et al. MicroRNA-203 inhibits long noncoding RNA HOTAIR and regulates tumorigenesis through epithelial-to-mesenchymal transition pathway in renal cell carcinoma. Mol Cancer Ther. 2018. https://doi. org/10.1158/1535-7163.mct-17-0925.

77. Kim K, et al. HOTAIR is a negative prognostic factor and exhibits prooncogenic activity in pancreatic cancer. Oncogene. 2013;32:1616-25. https://doi.org/10.1038/onc.2012.193.

78. Yang Z, et al. Overexpression of long non-coding RNA HOTAIR predicts tumor recurrence in hepatocellular carcinoma patients following liver transplantation. Ann Surg Oncol. 2011;18:1243-50. https://doi. org/10.1245/s10434-011-1581-y.

79. Zheng J, et al. Curcumin up-regulates phosphatase and tensin homologue deleted on chromosome 10 through microRNA-mediated control of DNA methylation - a novel mechanism suppressing liver fibrosis. FEBS J. 2014:281:88-103. https://doi.org/10.1111/febs.12574.

80. Yu F, et al. HOTAIR epigenetically modulates PTEN expression via MicroRNA-29b: a novel mechanism in regulation of liver fibrosis. Mol Ther. 2017;25:205. https://doi.org/10.1016/j.ymthe.2016.10.015.

81. Chen L, Li J, et al. Overexpression of LncRNA AC 067945.2 down-regulates collagen expression in skin fibroblasts and possibly correlates with the VEGF and Wnt signalling pathways. Cell Physiol Biochem. 2018;45(2):76171. https://doi.org/10.1159/000487167. 